\title{
Antibacterial Susceptibility Patterns of Common Bacterial Species associated with Urinary Tract Infections in Patients Attending Kam Medical and Diagnostic Centre, Kampala Uganda.
}

\author{
Gerald Turyatunga $^{a}, 1$, Sarah Gift Wito ${ }^{a}$, Enoch Muwagunzi ${ }^{\text {b }}$ \\ a Faculty of Health Sciences, University of Kisubi \\ ${ }^{b}$ Faculty of Medicine, Mbarara University of Science and Technology
}

Abstract

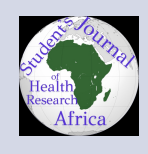

Background: ${ }^{a}$

Urinary tract infection (UTI) is defined as the presence of microbial pathogens within the urinary tract. It is primarily caused by Escherichia coli (E.coli), accounting for $75 \%$ of all bacterial UTI cases. Other bacteria such Klebsiella pneumonia, Proteus mirabilis, Staphylococcus aureus, Pseudomonas aeruginosa, and Enterococcus faecalis have also been reported as causative agents. The study aimed to determine the antibiotic susceptibility patterns of Uropathogenic bacteria in urine samples of patients with suspected UTI in Kam Medical and the diagnostic Centre.

Methodology:

This was a cross-sectional study where 120 urinary samples from Kam Medical and Diagnostic Centre in 2019. The urine specimens were cultured on CLED (Cysteine Lactose Electrolyte - Deficient) and blood agar media. Kirby-Bauer's standard disk diffusion method was applied to test the susceptibility of the drug for Mueller-Hinton culture agar plates. Results:

All 120 patients suspected of UTI had bacterial pathogen causing UTI. Among the urinary pathogens, Escherichia coli was the most common in 85/120 (70.8\%) of the patients followed by S.aureus 13/120 (10.8\%), Klebsiella spp 4/120 (9.2\%), Enterococcus spp with 4/120 $3.3 \%$ ), Pseudomonas aeruginosa with 4/120 (3.3\%) and Proteus with 3/120 (2.5\%). According to the results of the antibiogram, the highest resistance was observed for Nalidixic acid $(64.2 \%)$, Ampicillin (61.7\%), and Cotrimoxazole (54.2\%). The highest susceptibility (antibiotic sensitivity) was observed with imipenem (97.5\%), Nitrofurantoin (49.2\%), Ciprofloxacin (45.8\%), and Clotrimazole (44.2\%)

Conclusion and recommendations:

The bacterial pathogens associated with UTIs in this study were E.coli species, Staphylococcus aureus, Klebsiella, Enterococcus species, Pseudomonas species, and Proteus species. E.coli was the most common isolate followed by S.aureus, Klebsiella spp, Pseudomonas spp, and Enterococcus spp, and lastly Proteus spp. The highest levels of bacterial resistance were recorded against first-generation antibiotic drugs The bacterial isolates in this study were highly susceptible to broad-spectrum, second/ third generation antibiotics drugs.

\footnotetext{
${ }^{a}$ email: turlatungager-

ald2014@gmail.com date submitted:

9th/02/2021 date accepted: 21st/03/2021
} 


\section{Background}

Urinary tract infection (UTI) is defined as the presence of microbial pathogens within the urinary tract Muthulakshmi et al., (2017). It is primarily caused by Escherichia coli (E.coli), accounting for $75 \%$ of all bacterial UTI cases. Other bacteria such as Klebsiella pneumonia, Proteus mirabilis, Staphylococcus aureus, Pseudomonas aeruginosa, and Enterococcus faecalis have also been reported as causative agents (Antwi et al., 2008; Boye et al., 2012).

UTI can be classified based on the site of infection; Infection of the bladder is known as cystitis, and infection of the kidneys is called pyelonephritis (Flores-Mireles et al., 2015). It can also be classified clinically as either complicated or uncomplicated depending on the extent of infection (Bennett et al., 2014). UTIs are highly intricate and are difficult to treat. Complicated urinary tract infections can lead to structural abnormalities that lower the ability of the urinary tract to flush out the urine hence bacteria are provided with better scope for growth (Sabih et al., 2021).

Globally, UTIs are a major public health burden leading to increased morbidity and the associated high healthcare cost (Flores-Mireles et al., 2015). According to World Health Organization (WHO), UTIs are responsible for 8.3 million hospital visits and more than 1 million hospitalizations leading to an overall annual cost of over $\$ 1$ billion (WHO, 2013). Worldwide, it is evident that urinary tract infections are more common among young girls because women have a shorter urethra as compared to men (Odoki et al., 2019) show that an estimated $50 \%$ of women report having had a UTI at some point in their lives. In Uganda, the prevalence of UTI was reported to be $13.3 \%$ by Odoki et al., (2019). In Tanzania, a study conducted on patients in the pediatric ward of Muhimbili Hospital and Mulago National Referral Hospital in Uganda between five to ten years reported prevalence of UTI to be $16.8 \%$ and $14.6 \%$ respectively.

Antibiotics play an important role in minimizing morbidity and mortality associated with UTIs. The most common antibiotics used for the treatment of UTI are cefuroxime; amoxicillin/clavulanic acid, trimethoprim/sulphamethoxazole, and fluoroquinolones. However, in Ugandan clinical practice, there is the unorthodox treatment of patients suspected of UTI, typically patients have blindly prescribed antibiotics without valid microbial culture and sensitivity laboratory results. This affects the efficacy of the treatments due to the rise of antimicrobial-resistant bacteria which leads to treatment failure, hence increased morbidity and ultimately high cost of treatment (Uganda Clinical Guidelines, 2016). One way to avert this problem is detailed knowledge of the prevalence of bacteria causing UTI and the antibiotic susceptibility pattern. Thus, this study will investigate the prevalence and antibiotic susceptibility pattern of Uropathogenic bacteria isolated from urine samples of patients with suspected UTI, in Kam Medical and Diagnostic center Kampala, Uganda.

The rise of antimicrobial-resistant bacteria (AMR) is a global public health concern because it leads to treatment failure, hence increased morbidity and mortality. It also leads to higher treatment costs, therefore, placing a greater burden on the economy. This rise is partly driven by the blind indiscriminate use of antibiotics. In Uganda suspected UTI patients have blindly prescribed antibiotics without valid microbial culture and sensitivity laboratory results and this contributes to treatment failure. Secondly, most patients with suspected UTI, out of ignorance visit local drug shops often run by under-trained medical personnel who sell antibiotics drugs for treatment without any prescriptions, further leading to treatment failure and antibiotic resistance.

Despite this, in sub-Saharan Africa, for example, Uganda, where bacterial infections are common and there is a paucity of information on the common causes of UTIs and antibiotic susceptibility patterns of the causative species.

Given the above limitations, this study will elucidate the bacterial pathogens commonly associated with UTIs at KAM Medical and Diagnostic Centre and the antimicrobial susceptibility patterns to aid successful prognosis.

\section{Materials And Methods}

\section{Study area}

The study was carried out in the Microbiology (bacteriology) department of the Kam Medical and Diagnostic Centre. Kam medical is a private medical center with four departments in the Kawempe division Kampala, Uganda. It lies between Mulago National Referral hospital and Kubiri roundabout Kalerwe road. The Kam Medical and diagnostic Centre is a newly constructed medical center and 
started in 2015 in the Kawempe division. It caters to people living in and around Kampala capital city.

\section{Study design}

This was a cross-sectional study where 120 patients presenting with signs and symptoms for UTI attending Kam medical and diagnostic center were purposively sampled.

\section{Sample size}

The sample size was determined using the Kish and Lisle formula (1965) for cross-sectional studies.

$$
n=z 2 p(1-p) / d 2
$$

Where: $z=Z$ score for $95 \%$ confidence interval $=$ 1.96, $p=$ prevalence, $d=$ tolerable error $=5 \%$. Where $z=10, p=6, d=5 \% .=120$. Therefore 120 samples were collected and analyze.

\section{Sample collection and analysis}

After patients had given consent, $20 \mathrm{ml}$ midstream urine was collected into a sterile screwcapped, universal container and the sample containers were labeled with patient details such as name, age, laboratory number, and sex. The labeled specimens were transported to the laboratory and processed within an hour of collection. A $1.5 \mathrm{ml}$ aliquot of the sample was placed into a sterile cryovial labeled with patient information for microbiological analysis.

\section{Urine dipstick}

For biochemical analysis, urine samples were analyzed using the 10 parameter urine test strips. Each strip was dipped in the urine sample and analyzed according to the manufacturer's instructions. The results for the biochemical analysis were recorded awaiting culture and sensitivity results.

\section{Microbiological culture}

Cysteine Lactose Electrolyte - Deficient (CLED), blood agar was prepared using the manufacturer's instructions (Rosco Diagnostic). 15-20 ml CLED agar was poured into a sterile Petri dish (90- 100 $\mathrm{mm}$ diameter), allowed to solidify, and incubated at $37^{\circ} \mathrm{C}$ for 18 to 24 hours. After incubation, the plates were checked for sterility then inoculated with $50 \mu$ l of the sample stored in the $1.5 \mathrm{ml}$ cryovial. The samples were inoculated using the streak plate method and then incubated for 18 to 24 hours at $37^{\circ} \mathrm{C}$ for growth to take place.

Isolation and identification of bacterial isolates

Following 24 hours of incubation, the plates were inspected for bacterial growth. Bacterial colony numbers less than 104/ml were considered to be insignificant and possible contamination. For colony growths considered to be significant, the bacterial colonies were further identified using colony morphology, gram reaction, and biochemical tests.

\section{Colony morphology}

The appearances of urinary pathogens on CLED agar as described by Cheesborough, 2006 was used for the primary identification of the bacterial colonies.

E. coli appear as Yellow (lactose-fermenting) opaque colonies often with a slightly deeper colored center and S.aureus appear as deep yellow colonies of uniform color.

Klebsiella species appear as large mucoid yellow or yellow-white colonies and Proteus species appear as translucent blue-grey colonies.

Aeruginosa appears as Green colonies with rough periphery (characteristic color and

Faecalis appears as Small yellow colonies

Saprophyticus and other coagulasenegative staphylococci appear as yellow to white colonies.

\section{Gram stain technique}

Smears of the bacterial colonies were made and analyzed using the gram staining technique and classified as gram-negative or gram-positive based on the appearance. Gram-positive bacteria stained dark purple and gram-negative bacteria stain red. Following gram staining, gram-negative bacteria such as E. coli, Klebsiella spp., Proteus mirabilis, Pseudomonas spp., Enterococcus faecalis, and S. aureus was confirmed using the following biochemical tests: indole, citrate, and urea. Pseudomonas aeruginosa was confirmed using the cytochrome oxidase test. For the gram-positive bacteria, the bacterial colonies were confirmed using the catalase and coagulase test.

\section{Catalase test}

This test as described by Cheesborough, 2006 was used to differentiate suspected Staphylococci species from streptococci. Two drops of $3 \%$ hydrogen peroxide were put onto a sterile glass slide using a sterile Pasteur pipette. A gram-positive yellow colony suspected to be either Staphylococcus or S treptococci was picked using a sterile wooden applicator stick and placed in the hydrogen peroxide on the glass slide. If bubbling was observed the organism was considered catalase-positive and confirmed as Staphylococcus sp. If no bubbling 
was observed the organism was considered to be catalase-negative and possibly as Streptococcus.

\section{Free coagulase test}

Bacterial colonies that were confirmed to be Staphylococcus spp were further differentiated into

S. aureus (pathogenic) from S. albus (nonpathogenic) using the coagulase test as described by WHO, 2013. A drop of distilled water was placed on a slide and the test colony was added and mixed to make a suspension. A drop of plasma was added to the suspensions and mixed gently. The mixture was observed for clumping after 10 seconds. If clamping was observed, the colony confirmed was as S. aureus.

\section{Oxidase test}

Bacterial colonies which appeared Green with the rough periphery and suspected to be P.aeruginosa were subjected to the oxidase test for species confirmation. A filter paper was placed in a sterile Petri dish and 2 drops of oxidase reagent were added. Using a sterile applicator stick, a colony of the test organism was picked and transferred onto the filter paper where oxidase reagent was placed. After a few seconds, the filter paper was observed for the development of a blue-purple color. If the Blue-purple color was observed the test was considered to be oxidase-positive and the bacterial colony was confirmed as Pseudomonas spp (WHO, 2013).

\section{Indole}

This test was used in organisms suspected to be $E$. coli and Klebsiella. Indole test determines the presence or absence of the tryptophanase, an enzyme that breaks down tryptophan (WHO, 2013). $1 \mathrm{ml}$ of $1 \%$ Tryptone broth mixed with one suspected colony and the mixture was incubated for 12 to 18 hours. After incubation Kovac's reagent was added to the Tryptone/sample mixture, the development of the red ring at the interface was read as a positive result for E.coli and Klebsiella (Colony morphology was used for differentiation of E.coli and Klebsiella)

\section{Citrate test}

Small yellow colonies that tested gram-positive were suspected as Enterococcus and subjected to the citrate utilization test. Citrate test was used to test for the presence of citrate which is the sole source of carbon for bacteria (WHO, 2013). An agar slant with a synthetic medium containing small amounts of mineral salts (citrate and ammonium) was used to perform the test (WHO, 2013). Bromothymol blue ( $\mathrm{pH}$ indicator) was added to the agar slant. Using a sterile wire loop, colonies of suspected Enterococcus were inoculated in the citrate agar and then incubated aerobically at $37^{\circ} \mathrm{C}$ for 12 to 18 hours. The media was then observed for color change. A blue color was interpreted as positive for Enterococcus spp.

\section{Antimicrobial susceptibility testing}

Each bacterial isolate was subjected to antimicrobial susceptibility testing using the standard Kirby Bauer's disc diffusion method (Bauer et al., 2012). A sterile wire loop was used to obtain a loop full of the sample and the sample was then streaked over the Mueller Hinton agar evenly to form a surface. Antibiotic discs of Ciprofloxacin $(5 \mu \mathrm{g})$, Nalidixic acid $(30 \mu \mathrm{g})$, Nitrofurantoin $(30 \mu \mathrm{g})$, Cotrimoxazole $(25 \mu \mathrm{g})$, Ampicillin (30 $\mu \mathrm{g}$.) and imipenem $(30 \mu \mathrm{g})$, were placed onto each plate and equally spaced out using a disc dispenser. The plates were then incubated at $37^{\circ} \mathrm{C}$ for 18 hours. The Zones of inhibition were measured using a ruler to the nearest millimeter and compared to the standards provided by the CLSI (2009). The zones were interpreted as susceptible, intermediate or resistant.

\section{Quality control}

Standard reference Control strains of Staphylococcus aureus ATCC 25923, Escherichia coli ATCC 25922.,Pseudomonas aeruginosa ATCC 27853 recommended by the National Committee on Clinical Laboratory Standards (2004) was used for the quality control test.

The samples were placed sterile universal container. Isolation and culturing were done under maximum aseptic conditions like Petri dishes; media, wire loops, and forceps were all sterilized before use. The work surfaces were disinfected with $70 \%$ alcohol before and after work. All plates were checked for sterility before use by incubating at $37^{\circ} \mathrm{C}$ then overnight and checking for any bacterial growths or contamination.

\section{Inclusion criteria}

Patients presenting with signs and symptoms of urinary tract infection as indicated by the clinician and not on antibiotic therapy were included in the study.

\section{Exclusion criteria}

Patients presenting with signs and symptoms of urinary tract infection but who were on antibiotic therapy were not included in the study. 


\section{Data analysis}

Patients' identification names were not used, numbers and letters were used to label the samples. The information was entered into excel spreadsheets before being analyzed using Microsoft excel. The occurrence of UTIs caused by microbial uropathogens was calculated using Microsoft excel for microbiological analysis.

\section{Ethical clearance}

Clearance for the study was obtained from the medical superintendent of Kam Medical and Diagnostic centre.

Written informed consent was obtained from the patients before enrolling them into the study. For patients below 10 years, consent was granted by the parents or guardians. Patients were given unique identification numbers to provide confidentiality. These unique IDs were only known to the researcher and results were issued in person to the patients enrolled in the study.

\section{RESULTS}

\section{Demographic of patients enrolled in the study}

A total of 120 patients were enrolled in the study. Of these 97 were female and 23 were male. The youngest participant enrolled in the study was 1 year old and the oldest participant was 78 years old.

\section{Number of patients enrolled in the study}

The occurrence of urinary tract infection based on sex and age group in patients presenting with signs and symptoms at Kam medical and diagnostic center

All patients recruited in the study were confirmed positive for UTI using microbiological culture (Table 2).

\section{Bacterial species isolated from patient urine} samples

Bacterial species were isolated and identified using morphological appearance and biochemical tests. Of the bacterial species isolated, four were Gram-negative (E.coli spp, Klebsiella spp, Proteus spp., Pseudomonas spp., ) and two were Gram-positive (Enterococcus spp. and Staphylococcus aureus). E.coli was the most common bacteria isolated in 85 of the 120 patients recruited in the study followed by S.aureus (13/120), Klebsiella spp (11/120) Pseudomonas spp and Enterococcus spp 4/120, lastly Proteus species 3/120. This is shown in table 3

\section{Antibiotic susceptibility pattern of isolated bacteria}

The $E$. coli isolates were susceptible to imipenem followed by ciprofloxacin (Cipro), Nitrofurantoin (F), Nalidixic acid (NA), Clotrimazole (CXM), and lastly Ampicillin (Amp). The Klebsiella spp isolates in the study were susceptible to imipenem followed by ciprofloxacin, Nitrofurantoin, and lastly Ampicillin; S. aureus isolates were susceptible to Cotrimoxazole, imipenem, and Ampicillins. P. aeruginosa was more susceptible to imipenem followed by Clotrimazole Nalidixic acid, Ampicillin, and lastly Nitrofurantoin. Proteus spp was more susceptible to imipenem followed by Clotrimazole and lastly Nitrofurantoin. P. aeruginosa and Proteus spp were mostly susceptible to imipenem followed by Ampicillin, and ciprofloxacin.

\section{Discussion}

This study recruited 120 patients (97 female and 23 male) presenting with signs and symptoms of UTIs. All study participants (120/120) were confirmed positive for UTI using the microbiological culture of urine samples. The colonies from microbiological culture were subjected to morphological examination and biochemical tests to identify the bacterial species.

The most common bacterial species associated with UTIs isolated from patients in the study patients was E.coli in 85/120 patients followed by S.aureus (13/120), Klebsiella spp (11/12), Pseudomonas spp, and Enterococcus spp (4/120), and lastly Proteus spp (3/120). The findings of this study are similar National Institute for Health and Clinical Excellence-NICE (2012) where the common isolates associated with UTIs were E. coli, S. aureus, Klebsiella spp, Pseudomonas spp, Enterococcus spp., and Proteus spp. These bacteria belong to the group Enterobacteria which typically are considered to be part of the gut flora and due to improper hygiene practices, there is a great risk of cross-contamination of the urinary tract with bacteria from the gastrointestinal (Gl) tract. Although these bacteria are nonpathogenic in the GIT when they cross to the urinary tract they become pathogenic and cause discomfort and disease (Gupta et al., 2012). S.aureus a normal flora on the skin was also identified as a causative agent of UTI in 13/120 patients because when $S$. aureus crosses from the skin to the uri- 
Table 1. Demographic of patients enrolled in the study

\begin{tabular}{ll}
\hline Sex & Number of patients enrolled (percentage) \\
Males & $23(19.2 \%)$ \\
Female & $97(80.8 \%)$ \\
Total & $120(100 \%)$ \\
\hline
\end{tabular}

Table 2. Urinary Tract infection status among the age group that presented atKam Medical and diagnostic centre

\begin{tabular}{lll}
\hline Age Group & Number of patients & $\begin{array}{l}\text { Number of patients confirmed with UTI } \\
\mathbf{0}-\mathbf{1 0}\end{array}$ \\
$\mathbf{1 1} \mathbf{2 0}$ & 18 & $18 / 7(100 \%)$ \\
$\mathbf{2 1}-\mathbf{3 0}$ & 43 & $43 / 43(100 \%)$ \\
$\mathbf{3 1 - 4 0}$ & 21 & $21 / 21(100 \%)$ \\
$\mathbf{4 1 - 5 0}$ & 13 & $13 / 13(100 \%)$ \\
$\mathbf{5 1}-\mathbf{6 0}$ & 11 & $11 / 11(100 \%)$ \\
$\mathbf{6 1}-\mathbf{7 0}$ & 4 & $4 / 4(100 \%)$ \\
$\mathbf{7 1}-\mathbf{8 0}$ & 3 & $3 / 3(100 \%)$ \\
Total & $\mathbf{1 2 0}$ & \\
\hline
\end{tabular}

Table 3. Etiological bacterial agents isolated from patients confirmed with UTI

\begin{tabular}{lll}
\hline Organism Isolated & Frequency & Percentage \\
E coli & 85 & $70.8 \%$ \\
Klebsiella spp. & 11 & $9.2 \%$ \\
S. aureus & 13 & $10.8 \%$ \\
Pseudomonas spp. & 4 & $3.3 \%$ \\
Proteus spp. & 3 & $2.5 \%$ \\
Enterococcus spp. & 4 & $3.3 \%$ \\
Total & $\mathbf{1 2 0}$ & $\mathbf{1 0 0} \%$ \\
\hline
\end{tabular}

Table 4. Isolated Bacterial pathogenssusceptability patterns to antibiotic

\begin{tabular}{llllllll}
\hline Bacterial spp & Susceptibility & Cipro & NA & CXM & MEM & AMP & F \\
E.coli spp. & S & 43 & 35 & 35 & 83 & 31 & 40 \\
& $\mathrm{R}$ & 40 & 50 & 48 & 2 & 54 & 10 \\
Pseudomonas spp. & $\mathrm{I}$ & 2 & 0 & 2 & 0 & 0 & 29 \\
& $\mathrm{~S}$ & 0 & 1 & 1 & 4 & 1 & 2 \\
Klebsiella spp. & $\mathrm{R}$ & 4 & 3 & 3 & 0 & 3 & 0 \\
& $\mathrm{I}$ & 0 & 0 & 0 & 0 & 0 & 9 \\
S.aureus spp. & $\mathrm{R}$ & 6 & 0 & 5 & 10 & 2 & 2 \\
& $\mathrm{I}$ & 5 & 11 & 6 & 1 & 9 & 0 \\
& $\mathrm{~S}$ & 0 & 0 & 0 & 0 & 0 & 9 \\
& $\mathrm{R}$ & 1 & 6 & 9 & 13 & 8 & 3 \\
\end{tabular}


Table 5. Isolated Bacterial pathogenssusceptability patterns to antibiotic

\begin{tabular}{llllllll}
\hline Proteus spp. & $\mathrm{I}$ & 6 & 0 & 0 & 0 & 0 & 5 \\
& $\mathrm{~S}$ & 2 & 0 & 1 & 3 & 0 & 2 \\
Enterococcus spp. & $\mathrm{R}$ & 1 & 3 & 2 & 0 & 3 & 0 \\
& $\mathrm{I}$ & 0 & 0 & 0 & 0 & 0 & 1 \\
& $\mathrm{~S}$ & 3 & 1 & 2 & 4 & 4 & 3 \\
& $\mathrm{R}$ & 1 & 3 & 2 & 0 & 0 & 0 \\
& $\mathrm{I}$ & 0 & 0 & 0 & 0 & 0 & 1 \\
\hline
\end{tabular}

nary tract it is capable of causing a urinary tract infection.

UTI's can be caused by one or more bacteria, and in this study, there were no co-infections reported. Typically co-infections are associated with hospitalacquired infection (HAl) and immune-suppressed persons (Abu-Bakr, 2010).

The susceptibility profiles of the 120 bacterial isolates were tested against six commonly used antibiotics. The highest resistance was observed with Nalidixic acid (77/120) followed by Ampicillin (74/120) and Cotrimoxazole (65/120) regardless of bacterial species. The findings of this study are similar to the study by Antwi et al., (2018), who similarly reported the highest resistance with Nalidixic acid (31.1\%), Ampicillin (58.6\%), and Cotrimoxazole (44.6\%). These are first-line antibiotics and are often cheap and readily available hence are often misused and this leads to the emergence and spread of antibiotic resistance. Most of the isolates were susceptible to imipenem (117/120) followed by Nitrofurantoin (59/120) and ciprofloxacin (55/120). Imipenem and Nitrofurantoin are betalactam antibiotics that have potent activity against a wide range of Gram-positive and Gram-negative bacteria. Ciprofloxacin a second-generation broadspectrum antibiotic of the fluoroquinolones class is costly in Uganda. Due to this ciprofloxacin is rarely misused hence it remains effective in the treatment of several bacterial infections. Imipenem a broadspectrum beta-lactam antibiotic was also effective against most of the bacterial isolates. Imipenem was recently introduced to the Ugandan market and is expensive relative to Uganda's income per capita therefore it is rarely prescribed and hence it is not misused and abused. The finding of this study is comparable to the study by Antwi et al., (2018), who reported the highest susceptibility to imipenem (24.3\%) and Nitrofurantoin (21.9\%).
For the bacterial species isolated in this study, antibiotic resistance was highest in E.coli. The E.coli isolates were highly resistant to Nalidixic acid (64.9\%) followed by Cotrimoxazole (73.8), Ampicillin (72.9\%). However, the isolates were susceptible imipenem (2.5\%) and Nitrofurantoin (12.5\%).

\section{Conclusions and Recommendations. 6 Conclusions}

The bacterial pathogens associated with UTIs in this study were E.coli species, Staphylococcus aureus, Klebsiella, Enterococcus species, Pseudomonas species, and Proteus species. E.coli was the most common isolate followed by S.aureus, Klebsiella spp, Pseudomonas spp, and Enterococcus spp, and lastly Proteus spp.

The highest levels of bacterial resistance were recorded against first-generation antibiotic drugs which include Nalidixic acid (64.2\%), Ampicillin (61.7\%), and clotrimazole (54.2\%).

The bacterial isolates in this study were highly susceptible to broad-spectrum, second/ third generation antibiotics drugs which include imipenem, Nitrofurantoin, and ciprofloxacin. Imipenem and Nitrofurantoin was the most potent antibiotic on the organisms tested. All isolates in this study were sensitive to imipenem.

\section{Recommendations}

Culture and sensitivity should be encouraged for proper diagnosis and identification of effective drugs to clear infections and hence avoid recurrence of UTIs. This will lead to improved efficacy of treatments and hence lower treatment costs. Furthermore, this will limit the emergence of new antibiotic-resistant bacteria.

Health workers and government officials should educate the public on the effect of self-medication 
and mobilize patients to ensure that they have duly completed the prescribed antimicrobial therapy because under dosage selects for resistance and as result will lead to treatment failure, increased treatment costs, and increased mortality due to lack of effective antibiotics to treat infections

\section{Acknowledgment}

I am greatly indebted to all staff at the Microbiology Laboratory at Kam Medical and Diagnostic Centre for their support. 
Table 6. LIST OF ABBREVIATIONS AND ACRONYMS

\begin{tabular}{ll}
\hline ASBA & Asymptomatic bacteriuria \\
CDC & Centre of disease control \\
CFU & Colony forming unit \\
CLED & Cysteine lactose electrolyte deficient Agar \\
KMDC & Kam medical and diagnostic Centre \\
RPM & Revolutions per minute \\
STI & Sexual transmitted infection \\
UNIK & University of Kisubi \\
UTI & Urinary tract infection \\
WHO & World Health Organization \\
MDR & Multi Drug Resistance \\
MR & Methyl red \\
MRSA & Multi-resistant Staphylococcus aureus \\
NCCLS & National committee for clinical laboratory standards \\
IMVIC & Indole test, Methyl red test, Voges-proskaur test and Citrate test \\
\hline
\end{tabular}




\section{Table 7. References}

1) Abu-Bakr, E.-M. M. (2010). Antimicrobial susceptibility pattern of pathogenic bacteria causing urinary tract infections at the Specialist Hospital, Yola, Adamawa State, Nigeria.

2) Aiyegoro, O. I. (2017). Incidence of urinary tract infections (UTI) among children and adolescents in Ile-Ife, Nigeria. . Afr J Microbial Res, 1, 13-19.

3) Antwi, S. B.-B. (2018). Urine dipstick as a screening test for urinary tract infection. Annals of Tropical Paediatrics: . International Child Health, 28(2)," 117-122. .https://doi.org/10.1179/146532808X302134

4) Boye, A. S. ( 2012). Asymptomatic urinary tract infections in pregnant women attending antenatal clinic in Cape Coast, Ghana. E3 J Med Res, 1(6), 74-83.

5) Flores-Mireles, A. L., Walker, J. N., Caparon, M., \& Hultgren, S. J. (2015). Urinary tract infections: epidemiology, mechanisms of infection and treatment options. Nature reviews. Microbiology, 13(5), 269-284. https://doi.org/10.1038/nrmicro3432

6) Gupta K., S. D. (2012). Antimicrobial resistance among uropathogens that cause community acquired urinary tract infections in women: a nationwide analysis. Clinically Infectious diseases, 33:89-94.https://doi. org/10.1086/320880

7) Muthulakshmi M., Gopalakrishnan S. (2017). Study on urinary tract infection among females of reproductive age group in a rural area of Kancheepuram district, Tamil Nadu. International Journal of Community Medicine and Public health. https://doi.org/10.18203/2394-6040.ijcmph20174274

8) National Institute for Health and Clinical Excellence-NICE.Urinary tract infection in children: diagnosis, t. a.-t. (2012). Urinary tract infection in children: diagnosis, treatment and long-term management. NICE clinical guideline. www.nice.org.uk/cg054. Accessed on 1ST September 2013.

9) Odoki M„ Adamu A. A., Tibyangye J., Nyabayo M. J., Wampande E., Kato C D., Agwu E., Bazira J., (2019). "Prevalence of Bacterial Urinary Tract Infections and Associated Factors among Patients Attending Hospitals in Bushenyi District, Uganda", International Journal of Microbiology, vol. 2019, Article ID 4246780, 8 pages, h ttps://doi.org/10.1155/2019/4246780https://doi.org/10.1155/2019/4246780

10) Sabih A, Leslie SW. Complicated Urinary Tract Infections. [Updated 2021 Feb 10]. In: StatPearls [Internet]. Treasure Island (FL): StatPearls Publishing; 2021 Jan-. Available from: https://www.ncbi.nlm.nih.g ov/books/NBK436013/

11) WHO. (2013). Manual for the laboratory identification and antimicrobial susceptibility testing of bacterial pathogens of public health importance in the developing world. USA. 